\title{
Surgery for Aneurysmal Right Coronary Fistula and Constrictive Pericarditis in an Adult: Case Report
}

\author{
Jaffar S. Shehatha ${ }^{1,2}$, Abdulsalam Y. Taha ${ }^{3 *}$ \\ ${ }^{1}$ Department of Cardiac Surgery, PAR Hospital, Irbil, Iraq \\ ${ }^{2}$ School of Surgery, Western Australia University, Perth, Australia \\ ${ }^{3}$ Department of Thoracic and Cardiovascular Surgery, School of Medicine and Sulaimania Teaching Hospital, \\ Sulaimania, Iraq \\ Email: salamyt1963@hotmail.com
}

Received 20 January 2014; revised 19 February 2014; accepted 12 March 2014

Copyright (C) 2014 by authors and Scientific Research Publishing Inc.

This work is licensed under the Creative Commons Attribution International License (CC BY). http://creativecommons.org/licenses/by/4.0/

(c) (i) Open Access

\begin{abstract}
Coronary artery fistula (CAF) is a direct communication between a coronary artery and the lumen of any of the cardiac chambers, i.e. the coronary sinus, the pulmonary artery, the superior vena cava or the proximal pulmonary veins. The majority of these fistulas are congenital in origin although they may occasionally be detected after cardiac surgery. Congenital CAF is a rare anomaly and aneurysmal formation in the fistula is even rarer. Majority of CAFs are isolated lesions, however, congenital or acquired heart diseases may co-exist. Herein, we report a case of huge congenital aneurysmal right CAF connected to the right atrium in an Iraqi man of 62 associated with tuberculous effusive-constrictive pericarditis to whom off pump pericardiectomy was performed followed by ligation of right coronary artery and vein graft implantation to its posterior descending branch under cardiopulmonary bypass. To the best of our knowledge, such association was not previously reported. CAF can be repaired surgically with minimum risk and excellent outcome. Surgery is advised whenever coronary fistula is diagnosed unless it is very small to avoid the potential complications.
\end{abstract}

\section{Keywords}

Coronary Artery Fistula; Ectasia; Aneurysm; Pericarditis; Pericardiectomy

\footnotetext{
${ }^{*}$ Corresponding author.
} 


\section{Introduction}

Coronary artery fistula (CAF) is a direct communication between a coronary artery (CA) and the lumen of any of the cardiac chambers, i.e. the coronary sinus, the pulmonary artery (PA), the superior vena cava (SVC) or the proximal pulmonary veins [1]. The majority of these fistulas are congenital in origin although they may occasionally be detected after cardiac surgery [2]. Congenital CAF is a rare anomaly and aneurysmal formation in the fistula is even rarer [1]. The angiographic incidence is around $0.1 \%$ to $0.2 \%$ [1] whilst the prevalence in the general population is $0.002 \%$ [3]. It was first described by Krause in 1865 [4]. Embryologically, congenital CAFs are thought to be due to incomplete closure of the sinusoids which normally connect the coronary arteries to the great vessels and chambers of the heart [4]. The fistula may involve the right (about 55\%) or the left (35\%) or both (5\%) coronary arterial systems [5]. It enters the right heart in about $90 \%$ of cases, thus producing a leftto-right shunt [5] but drainage into the left-sided chambers is less frequent [3]. The most common sites of drainage are the right ventricle (RV), followed by the right atrium (RA) and the PA [5]. They do not usually cause symptoms or complications in the first two decades, especially when small [2]. After this age, the frequency of both symptoms and complications increases [2]. The main differential diagnosis is patent ductus arteriosus, although other congenital arteriovenous shunts need to be excluded [2]. Coronary angiography is the main diagnostic tool [2]. Because of the severe complications that may develop due to coronary fistulas, every CAF deserves invasive treatment by either surgery or trans-catheter closure [3]. Herein, we report a case of huge congenital aneurysmal RCAF connected to RA in a man of 62 associated with tuberculous effusive-constrictive pericarditis. The management of this patient is presented with literature review.

\section{Case Summary}

A farmer of 62 consulted Irbil Centre for Cardiac Surgery (ICCS) on June 20th, 2009 because of progressive effort dyspnoea and chest pain of one year duration. He was a known case of effusive-constrictive tuberculous pericarditis diagnosed 8 months earlier. He had had two sessions of echocardiography-guided pericardiocentesis which proved this diagnosis followed by triple anti-tuberculous chemotherapy for 6 months. However, the drugs were not taken regularly and stopped when the patient felt a "complete" relief. He was admitted due to recent worsening of symptoms.

Physical examination revealed thin-looking, pale and dyspnoeic patient (New York Heart AssociationNYHA — class II-III) and a continuous parasternal murmur with prominent systolic component. There was an elevated jugular venous pressure (JVP) and mild leg oedema with slightly enlarged liver. Chest radiogram (CXR) showed cardiomegaly and pulmonary plethora. Electrocardiography (ECG) showed signs of inferior myocardial ischaemia whilst contrast-enhanced chest CT scan revealed a fistulous communication between the RCA and RA. Angiography showed a huge aneursymal RCA draining into the RA (Figure 1(a)-(c)), whereas the pressure measurements on cardiac catheterization were consistent with cardiac constriction.

Medications given to the patient included diuretic therapy besides the anti-tuberculous drugs. The patient was scheduled for surgery (pericardiectomy and repair of the aneurysmal RCAF).

Pericardiectomy: Median sternotomy was performed. The pericardium was then opened longitudinally; it was totally adherent to the heart. A small area of pericardium was removed by gentle dissection to expose the ascending aorta and RA wall to be ready for prompt aortic and caval cannulation and initiation of cardiopulmonary bypass (CPB) in case of any mishap. The procedure of pericardiectomy continued by gentle peeling of the thickened and calcified pericardium taking care not to injure the hugely dilated RCA. The LV was freed first and ultimately the heart surface between the two phrenic nerves was completely released "off bypass".

Repair of the Coronary Fistula: The fistula was located by careful palpation along the course of the RCA. The area of maximum thrill was over the RA; it was marked by a stitch in the nearby epicardial fat. Systemic heparinization was followed by aortic and bicaval cannulation. Then we went on total CPB and temperature reduced down to $32^{\circ} \mathrm{C}$. The dilated RA was then opened while the heart was still beating but to our surprise we failed to see the site of the fistula. Aortic cross clamp was then applied and cardioplegic solution given and again it was not possible to find the fistulous orifice. Lastly we elected to open the aneurysmal RCA longitudinally; but again we couldn't find the opening of the fistula due to the tortousity of the vessel. Therefore, and as a last resort, we decided to ligate the artery proximally near its origin and distally near the crus angle and implant a venous graft to posterior descending artery (PDA). When we came off bypass, the thrill had disappeared completely. The patient emerged from bypass smoothly. Haemostasis was secured. Closure of the wound was 


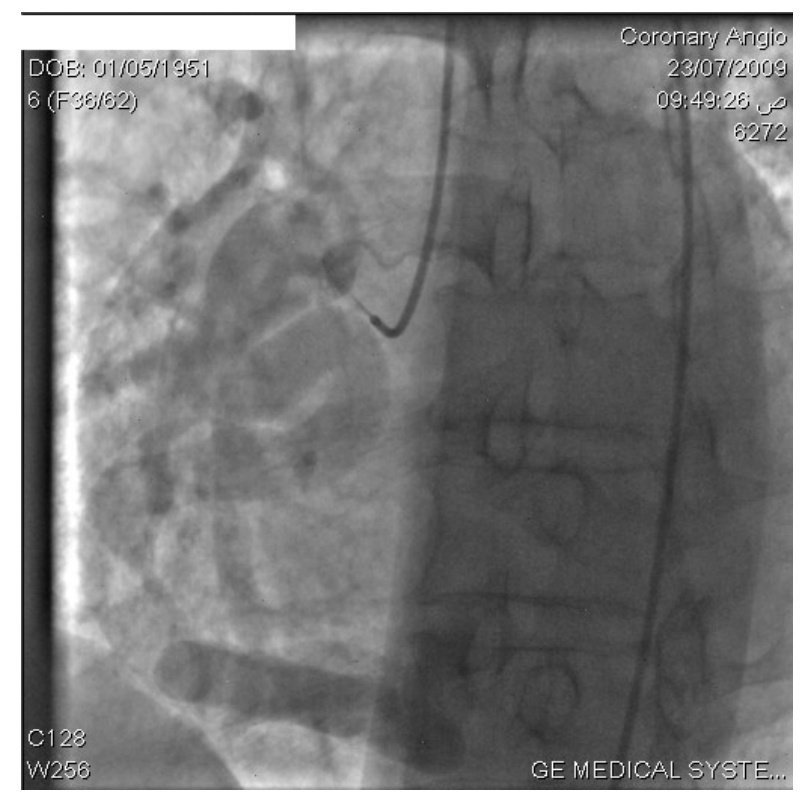

(a)

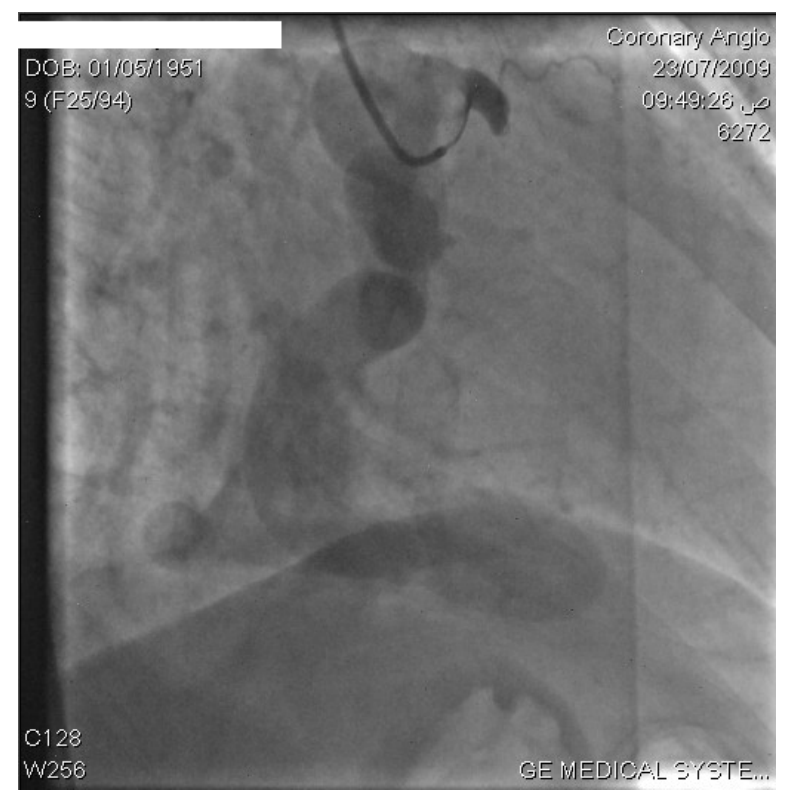

(b)

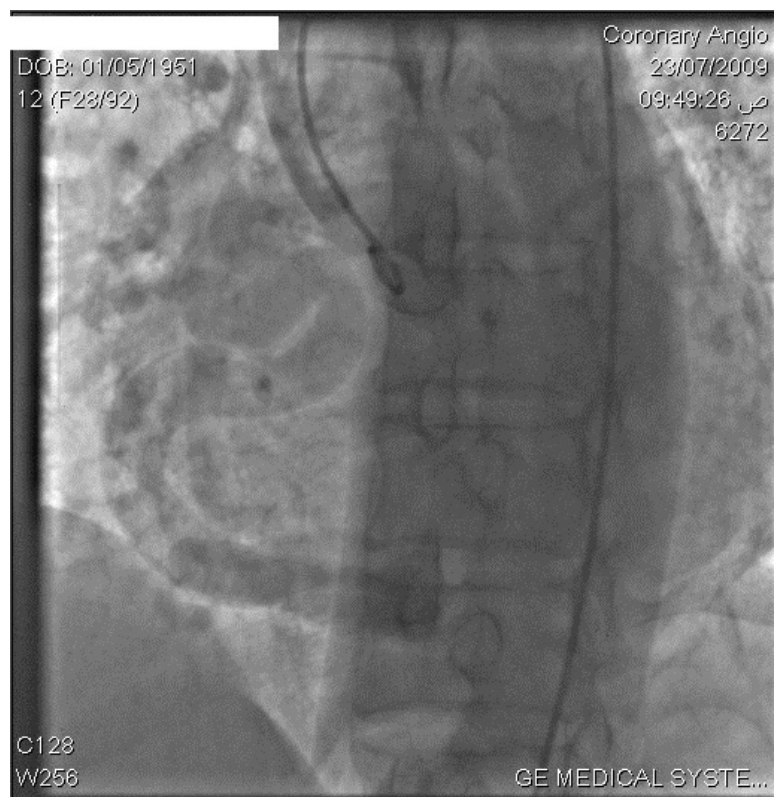

(c)

Figure 1. (a) Coronary angiogram showing aneurysmal RCA; (b) A second view of the aneurysmal RCF; (c) Aneurysmal RCF (third view).

done in the standard method.

The postoperative course was uneventful and the patient was discharged home on the 10th postoperative day on anti-tuberculous chemotherapy as the pericardial biopsy examination reconfirmed the diagnosis of tuberculous pericarditis. The patient was seen only once ( 2 years following surgery) and was in a very good health.

\section{Discussion}

Terms: Coronary artery ectasia or aneurysm is defined as a dilated coronary artery with a luminal diameter exceeding 1.5 times the diameter of the adjacent normal coronary segment or the largest diameter of the coronary artery. The dilatation is commonly diffuse and can be either fusiform or saccular in shape. A giant coronary 
aneurysm is usually defined as a coronary artery with a maximal diameter exceeding $20 \mathrm{~mm}$, although other cut off values $(50-150 \mathrm{~mm})$ have also been described. There are two types: atherosclerotic ( $>50 \%)$ and congenital coronary ectasia and aneurysm. The congenital variants are most commonly described in the RCA and are frequently associated with fistulas (as in our patient) [6].

Incidence: CAFs are rare. Al-Amiry et al. from Iraq studied 3000 adult patients who underwent diagnostic coronary angiography in 2 cardiac centers in Baghdad and found 4 patients with CAFs (0.13\%) [7]. Vigneswaran and Pollock from Ibn-Albitar hospital, Baghdad, Iraq in 1988 reported a case of left CA to PA fistula in a man of 41 associated with absent central PAs and ventricular septal defect [8]. The fistula was disconnected but construction of a modified Blalock-Taussig shunt failed [8]. This case could be the first and the only reported surgically treated CAF in Iraq. Our case therefore, might be the second.

Pathophysiology: Origin of the CAF can be any of the three major coronary arteries, including the left main trunk [3]. Characteristically, the CA that enters into anomalous communication with a cardiac chamber or the pulmonary trunk is obviously enlarged, not only dilated but elongated and tortuous [6] [9]. Coronary artery dilatation is common but degree of dilatation does not always depend on the shunt size [3]. In our case, though the exact size of RCA was not measured, it was huge comprising a giant aneurysmal vessel. Fistulas draining to right heart chambers, PA, coronary sinus or SVC constitute true arterio-venous communications whilst those opening into left side of the heart are actually arterio-arterial connections. When the fistula drains to the right side of the heart, the volume load is increased to the right heart as well as to the pulmonary vascular bed, the left atrium and the left ventricle. When the fistula drains into the left atrium or the left ventricle, there is volume overloading of these chambers but no increase in the pulmonary blood flow [2].

Clinical presentation: Nearly half of patients with fistulae are asymptomatic at diagnosis [4]. In our case, the patient was asymptomatic apart from the final year; when effort dyspnoea and chest pain developed most likely due to constrictive pericarditis. It is note-worthy that the cardiac murmur was never elicited during 61 years! The inferior myocardial ischemia noticed in our patient could be attributed to the steal effect of the coronary fistula [2] [4]. A thorough search on the net revealed no reported case of CAF associated with constrictive pericarditis. We think there was no causal relationship between the 2 conditions. However, this patient was very lucky not to have an injury to his aneurysmal RCA during the 2 sessions of diagnostic pericardiocentesis. Moreover, this extremely rare association added a technical challenge to the operation; the aneurysm could rupture at anytime during the tedious peeling of the pericardium.

The most consistent and striking physical sign: among reported cases is a continuous cardiac murmur [9]. The location of the maximal intensity of the continuous murmur seems to depend upon the site of the abnormal communication [9]. Neufeld et al. state that if a continuous murmur is localized in an area atypical for patent ductus arteriosus, the diagnosis of CAF should be suspected [9]. It deserves to note that when fistulas connect to the LV, only a diastolic murmur is anticipated [2] [9]. In one case reported by Scott D. H. there was no murmur; this is perhaps explained by the observation at necropsy that a thrombus had occluded the fistula [9]

Diagnosis: The diagnosis of CAFs is challenging as its prevalence is low, yet it should be considered in many symptomatic or asymptomatic patients presenting with cardiac murmurs [3]. Traditional way of diagnosis of CAF is invasive investigations such as cardiac catheterization and coronary angiography [3]. Other diagnostic modalities may be helpful like MRI and contrast-enhanced multislice tomography [3]. In our patient, CAF was evident on contrast-enhanced chest CT scan and further visualized by coronary angiography.

Management: The first surgical treatment of CAFs was done by Bjork and Crafoord in 1947 [3]. Nowadays, the treatment options for CAFs include surgery or catheter closure [2]. Some authors recommend closure of CAF even in asymptomatic patients to prevent fistula-related complications [3]. Surgery involves internal closure of the fistula within the receiving chamber or vessel (endocardial ligation) whenever feasible, but when the fistula is associated with a large aneurysm of the feeding artery, it may need to be ligated from within the aneurysm (epicardial ligation) [2] [3]. Surgery is associated with a low morbidity and mortality rate ranging from 0 to $6 \%$ [2].

Some authors have reported successful surgical occlusion of CAF on a beating heart without CPB [3]. This is usually feasible with simple and easily accessible fistulas [3] [5] at the end of a vessel [5]. In contrast, repair using CPB is preferred for lateral fistulas where significant areas of myocardium are perfused beyond the fistulas and also when aneurysmal dilatation is present [5].

Complications include thrombosis, distal embolization, myocardial ischemia and infarction, heart failure, arrhythmias, dissection, vasospasm, calcification, cardiac rupture and sudden cardiac death [6]. 


\section{Conclusion}

An adult patient with huge aneurysmal RCA to RA fistula and constrictive pericarditis is described. The patient lived symptom-free and his cardiac murmur, a common sign in CAF, was overlooked for almost 61 years. The inferior myocardial ischemia noticed in this patient could be attributed to the steal effect of the coronary fistula. Presenting symptoms in the final year were most likely caused by associated pericarditis. To the best of our knowledge, such association was not reported previously. The patient was lucky not to have an injury to the aneurysm during pericardiocentesis or surgery. Surgery was indicated for sake of constrictive pericarditis and repair of the fistula. The latter was done using CPB whilst pericardiectomy, which was completed on a beating heart. Though the fistulous orifice was not seen, exclusion of the aneurysmal RCA by proximal and distal ligatures abolished the fistula. Revascularization of distal RCA was necessary following this ligature.

\section{References}

[1] Darwazah, A.K., Hussein, I.H. and Hawari, M.H. (2005) Congenital Circumflex Coronary Arteriovenous Fistula with Aneurysmal Termination in the Pulmonary Artery. Texas Heart Institute Journal, 32, 56-59.

[2] Qureshi, S.A. (2006) Coronary Arterial Fistulas Review. Orphanet Journal of Rare Diseases, 1, 51. http://dx.doi.org/10.1186/1750-1172-1-51

[3] Ata, Y., Turk, T., Bicer, M., Yalcin, M., Ata, F. and Yavuz, S. (2009) Coronary Arteriovenous Fistulas in the Adults: Natural History and Management Strategies. Journal of Cardiothoracic Surgery, 4, 62. http://dx.doi.org/10.1186/1749-8090-4-62

[4] Ali, H.M., Clark, A.L., Caplin, J. and Bragadeesh, T. (2013) Congenital Circumflex Coronary Artery Fistulae; Presentation and Diagnosis: Case Report. BMJ Case Reports. http://dx.doi.org/10.1136/bcr-2012-006817

[5] Smith, A. and McKay, R. (2004) Chapter 28, Coronary Arterial Fistula. In: A Practical Atlas of Congenital Heart Disease [Internet], Springer-Verlag, London, 437. www.link.springer.com/content/pdf/10.1007\%2F978-1-85233-865-7.pdf

[6] Mazur, W., Siegel, M.J., Miszalski-Jamka, T. and Pelberg, R. (2013) Coronary Artery Anomalies. In: CT Atlas of Adult Congenital Heart Disease, Springer-Verlag, London, 136.

[7] Al-Amiry, H.A., Al-Najar, H.Y. and Bairam, A.R. (2008) Congenital Coronary Anomalies in Iraqi Adult Patients. Journal of Faculty of Medicine Baghdad, 50, 132.

[8] Vigneswaran, W.T. and Pollock, J.C.S. (1988) Pulmonary Atresia with Ventricular Septal Defect and Coronary Artery Fistula: A Late Presentation. Heart, 59, 387-388. http://dx.doi.org/10.1136/hrt.59.3.387

[9] Neufeld, H.N., Lester, R.G., Adams Jr., P., Anderson, R.C., Lillehei, C.W. and Edwards, J.E. (1961) Congenital Communication of a Coronary Artery with a Cardiac Chamber or the Pulmonary Trunk (“Coronary Artery Fistula”). Circulation, 24, 171-179. http://dx.doi.org/10.1161/01.CIR.24.2.171 